\title{
Leber's Hereditary Optic Neuropathy: a Case Report
}

\section{ART ICLE INFO}

\section{Article Type \\ Case Report}

\section{Authors}

Daneshvar Kakhki R.* MD
How to cite this article Daneshvar Kakhki R. Leber's Hereditary Optic Neuropathy: a Case Report. Horizon of Medical Sciences. 2017;23(2):157-159.
*Neurology Department, Medical School, Kashan University of Medical Sciences, Kashan, Iran

\section{Correspondence}

Address: Neurology Ward, Shahid Beheshtei General Hospital, Kilometer 5 of Qotb-e-Ravandi Boulevard, Kashan, Iran

Phone: $+98(31) 55345455$

Fax: $+98(31) 55345456$

redaneshvar@gmail.com

\section{Article History}

Received: June 28, 2016

Accepted: January 30, 2017

ePublished: March 25, 2017

\section{A B S T R A C T}

Aims One of the most prevalent mitochondrial genetic diseases is Leber disease. Most of the patients are with the bilateral ocular involvement, simultaneously in $25 \%$ and one after another in $75 \%$ of the patients with an average of 8-week involvement distance between two eyes. The aim of the study was to report a rare case of Leber disease with 11-year engagement distance between two eyes.

Patient Profile A 38 years old male patient, who had been hospitalized in autumn 2012 in Beheshti Hospital in Kashan, Iran, due to his left-eye vision loss, was studied. One day before referring to the hospital, his left-eye vision had gradually blurred, only could perceive light. The patient were with HBs Ag positive (Carrier), and was hypertensive from about one year before hospitalization. Based on the ophthalmoscopy results, the right-eye disc was lost and the left-eye disc was pale.

Findings At first, the patient received methylprednisolone pulse therapy, while no treatment response was observed after a 2-week therapy. Based on the genetic assessments of peripheral blood cells, the homoplastic 14233 mutation was diagnosed, confirming the clinical suspicion of Leber disease (LHON). The patient received 100mg a day Q10 coenzyme and multivitamin.

Conclusion The diagnosis of Leber hereditary optic neuropathy should be noticed in young men with bilateral optic atrophy.

Keywords Leber Hereditary Optic Neuropathy; Optic Neuritis; Mitochondrial DNA; Mutation

\section{CIT A T ION LIN KS}

[1] Ueber hereditäre und congenital-angelegte Sehnervenleiden [2] The epidemiology of pathogenic mitochondrial DNA mutations [3] Pedigree analysis in Leber hereditary optic neuropathy families with a pathogenic mtDNA mutation [4] Leber's hereditary optic neuropathy: The clinical relevance of different mitochondrial DNA mutations [5] Ophthalmologic findings in Leber hereditary optic neuropathy, with special reference to mtDNA mutations [6] Hereditary optic neuropathies [7] High incidence of visual recovery among four Japanese patients with Leber's hereditary optic neuropathy with the 14484 mutation [8] Alternative, simultaneous complex I mitochondrial DNA mutations in Leber's hereditary optic neuropathy [9] Clinical, genetic, and biochemical characterization of a Leber hereditary optic neuropathy family containing both the 11778 and 14484 primary mutations [10] Extensive investigation of a large Brazilian pedigree of 11778/haplogroup J Leber hereditary optic neuropathy [11] Heteroplasmy in Leber's hereditary optic neuropathy [12] Leber hereditary optic neuropathy: Does heteroplasmy influence the inheritance and expression of the G11778A mitochondrial DNA mutation? [13] Leber hereditary optic neuropathy: Clinical and molecular genetic findings [14] Mitochondrial mutations of Leber's hereditary optic neuropathy: A risk factor for multiple sclerosis [15] Multiple sclerosis and Leber's hereditary optic neuropathy mitochondrial DNA mutations [16] Mitochondrial DNA mutations in multiple sclerosis [17] Leber's hereditary optic neuropathy mitochondrial DNA mutations at nucleotides 11778 and 3460 in multiple sclerosis [18] Do idebenone and vitamin therapy shorten the time to achieve visual recovery in Leber hereditary optic neuropathy? [19] Variable pattern of visual recovery of Leber's hereditary optic neuropathy 
روز قبل از مراجعه ديد جشم جֶٍ بيمار به تدريج تار شده و يس إز از

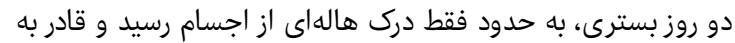

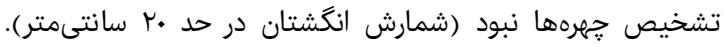

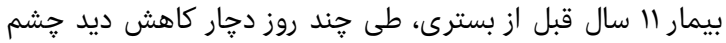

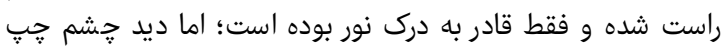

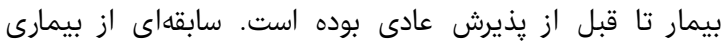

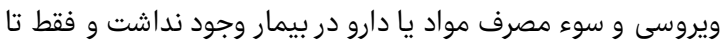

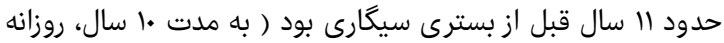

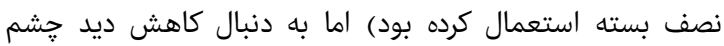

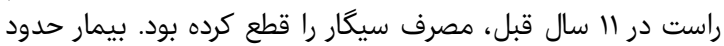

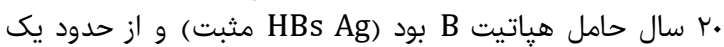

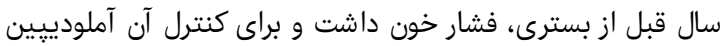

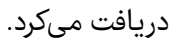
معاينات حشم بيمار حدت بينايى در حد درك نور در جشم راست و وران

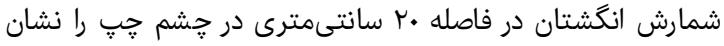

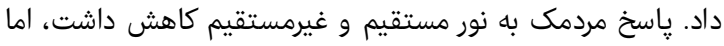

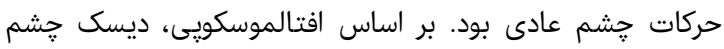

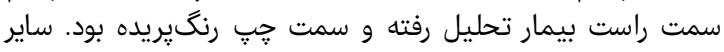

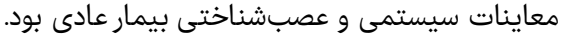

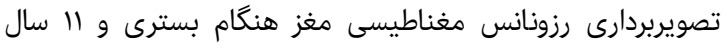

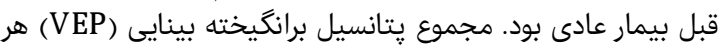

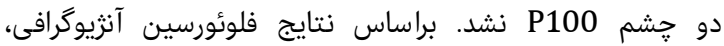

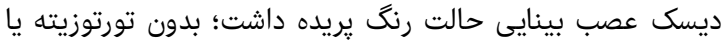

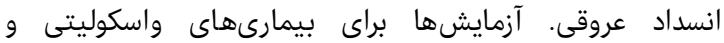

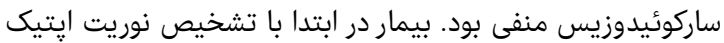

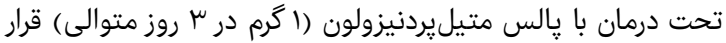

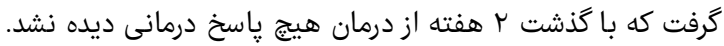

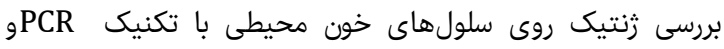

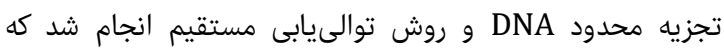

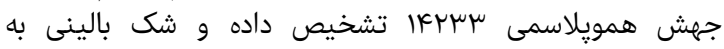

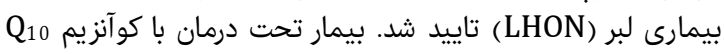

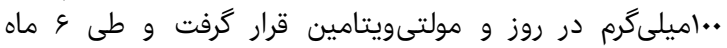

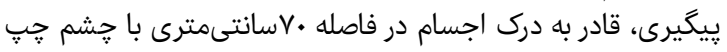

الكوى انتقال زنتيكى اين بيمارى اتوزومال غالب، اتوزومال

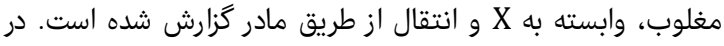

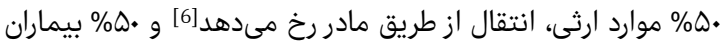

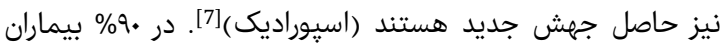

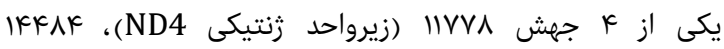

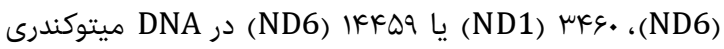

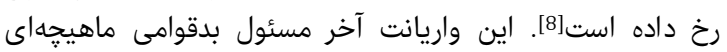

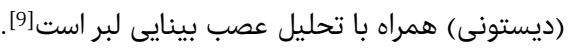

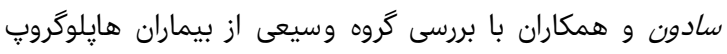

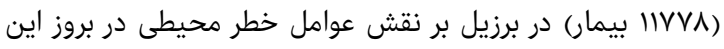

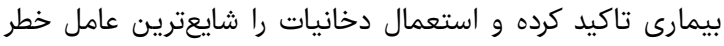

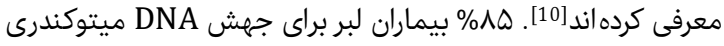

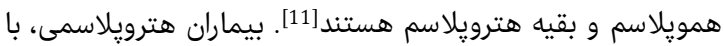

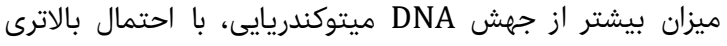

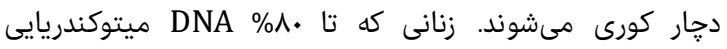

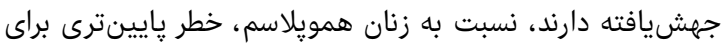
دوره سr، شماره r، بهار
نوروياتى ارثى عصب بينايى لبر؛ گزارش مورد

MD " رضا دانشور كاخكى

كروه نرولوزى، دانشكده يزشكى، دانشكاه علوم يزشكى كاشان، كاشان، ايران

קكيده

اهداف: بيمارى لبر، يكى از شايعترين بيمارىهاى زئنتيكى ميتوكندريايى است.

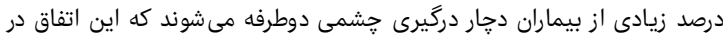

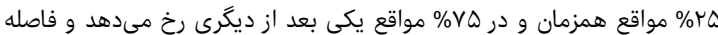

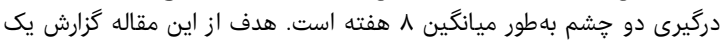

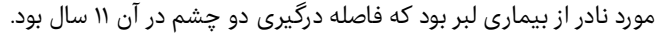

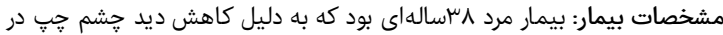

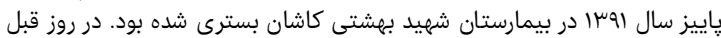

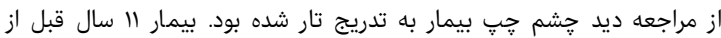

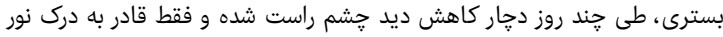

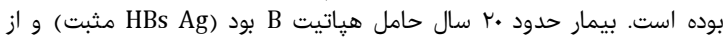

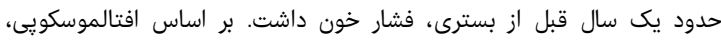

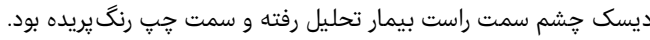

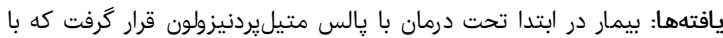

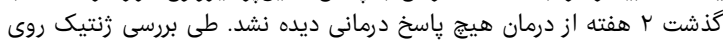

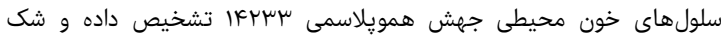

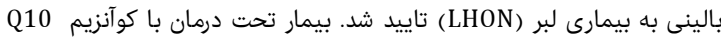

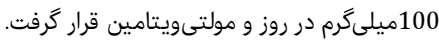

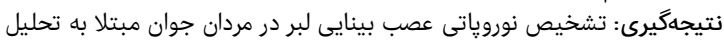

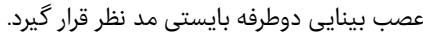

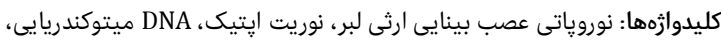

جهش كلروان

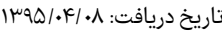

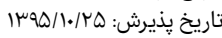
نويسنده مسئول: تاريخ: redaneshvar@gmail.com

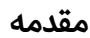

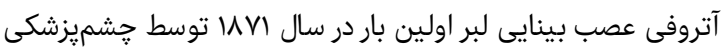

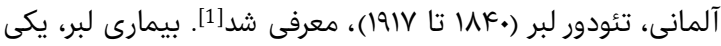

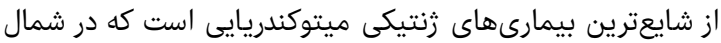

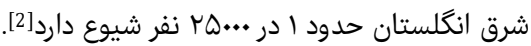

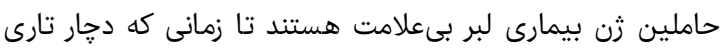

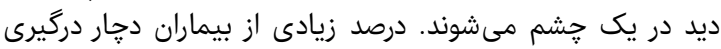

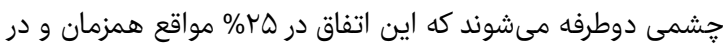

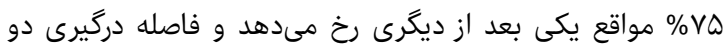

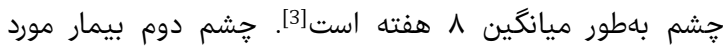

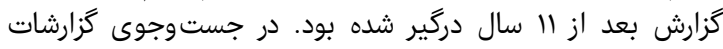

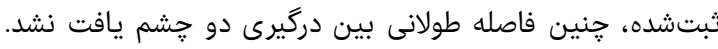

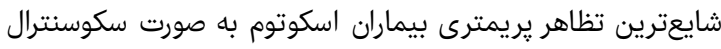

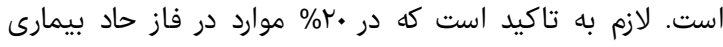

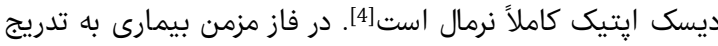

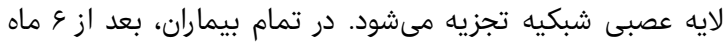

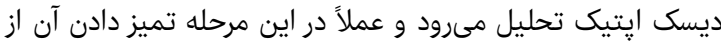

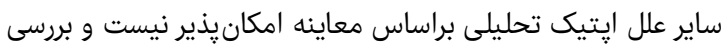

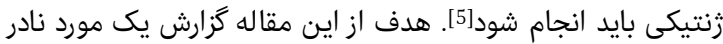

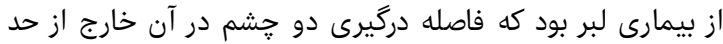

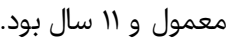

\section{مشخصات بيمار}

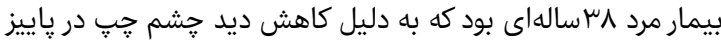

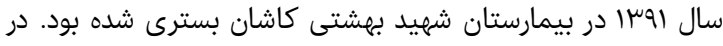

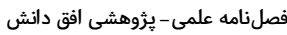


نوروياتى ارثى عصب بينايى لبر؛ گزارش مورد 109

families with a pathogenic mtDNA mutation. Am J Hum Genet 1995;57:77-86.

4- Riordan-Eva P, Harding AE. Leber's hereditary optic neuropathy: The clinical relevance of different mitochondrial DNA mutations. J Med Genet 1995;32(2):81-7.

5- Nikoskelainen EK, Huoponen K, Juvonen V, Lamminen T, Nummelin K, Savontaus ML. Ophthalmologic findings in Leber hereditary optic neuropathy, with special reference to mtDNA mutations. Ophthalmol. 1996;103(3):504-14.

6- Kerrison JB. Hereditary optic neuropathies. Ophthalmol Clin North Am. 2001;14(1):99-107.

7- Yamada K, Mashima Y, Kigasawa K, Miyashita K, Wakakura M, Oguchi Y. High incidence of visual recovery among four Japanese patients with Leber's hereditary optic neuropathy with the 14484 mutation. J Neuroophthalmol. 1997;17(2):103-7.

8- Johns DR, Berman J. Alternative, simultaneous complex I mitochondrial DNA mutations in Leber's hereditary optic neuropathy. Biochem Biophys Res Commun. 1991;174(3):1324-30.

9- Brown MD, Allen JC, Van Stavern GP, Newman NJ, Wallace DC. Clinical, genetic, and biochemical characterization of a Leber hereditary optic neuropathy family containing both the 11778 and 14484 primary mutations. Am J Med Genet. 2001;104(4):331-8.

10- Sadun AA, Carelli V, Salomao SR, Berezovsky A, Quiros PA, Sadun F, et al. Extensive investigation of a large Brazilian pedigree of 11778/haplogroup J Leber hereditary optic neuropathy. Am J Ophthalmol. 2003;136(2):231-8.

11- Smith KH, Johns DR, Heher KL, Miller NR. Heteroplasmy in Leber's hereditary optic neuropathy. Arch Ophthalmol 1993;111:1486-90

12- Chinnery PF, Andrews RM, Turnbull DM, Howell NN. Leber hereditary optic neuropathy: Does heteroplasmy influence the inheritance and expression of the G11778A mitochondrial DNA mutation?. Am J Med Genet. 2001;98(3):235-43.

13- Huoponen K. Leber hereditary optic neuropathy: Clinical and molecular genetic findings. Neurogenetics. 2001;3(3):119-25.

14- Vanopdenbosch L, Dubois B, D'Hooghe MB, Meire F, Carton H. Mitochondrial mutations of Leber's hereditary optic neuropathy: A risk factor for multiple sclerosis. J Neurol. 2000;247(7):535-43.

15- Pénisson-Besnier I, Moreau C, Jacques C, Roger JC, Dubas F, Reynier P. Multiple sclerosis and Leber's hereditary optic neuropathy mitochondrial DNA mutations. Rev Neurol (Paris). 2001;157(5):537-41. [French]

16- Kalman B, Lublin FD, Alder H. Mitochondrial DNA mutations in multiple sclerosis. Mult Scler. 1995;1(1):326.

17- Mojon DS, Herbert J, Sadiq SA, Miller JR, Madonna M, Hirano M. Leber's hereditary optic neuropathy mitochondrial DNA mutations at nucleotides 11778 and 3460 in multiple sclerosis. Ophthalmologica. 1999;213(3):171-5

18- Mashima Y, Kigasawa K, Wakakura M, Oguchi Y. Do idebenone and vitamin therapy shorten the time to achieve visual recovery in Leber hereditary optic neuropathy? J Neuroophthalmol. 2000;20(3):166-70.

19- Nakamura M, Yamamoto M. Variable pattern of visual recovery of Leber's hereditary optic neuropathy. Br J Ophthalmol. 2000;84(5):534-5
داشتن يسران مبتلا به لبر دارند[12]؛ با اين حال، هويونن اين

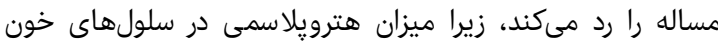

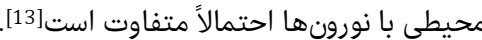

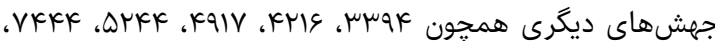

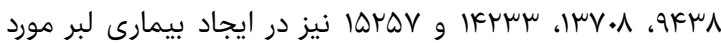
مطالعه قرار گرفته است[8]. احتمال همراهى بيمارىهاى خانواده MS

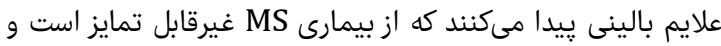

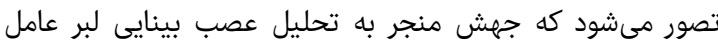

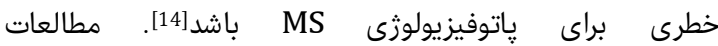
همهگيرىشناسى تاكنون نتوانستهاند در بيماران مبتلا به بـ

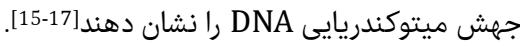

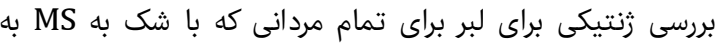

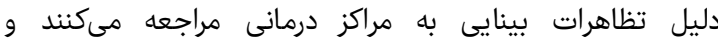

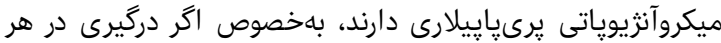

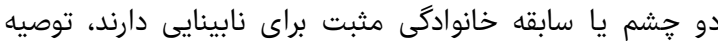

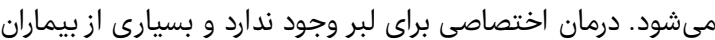

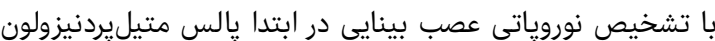

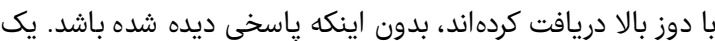

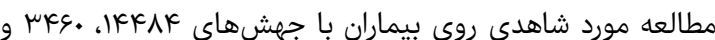
IIVVA بهبود در دسترس قرار گرفتن ATP مى شئنود، دريافت كردهاند، نشان

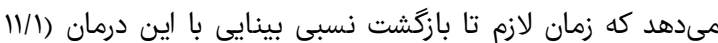

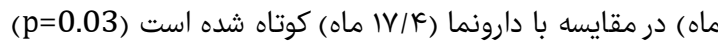

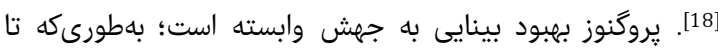

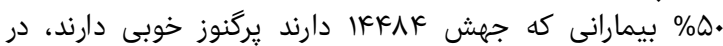

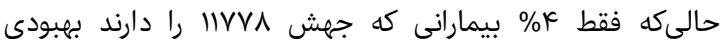

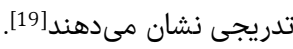
نتيجهكيرى

تشخيص نوروياتى عصب بينايى لبر در مردان جوان مبتلا به تحليل

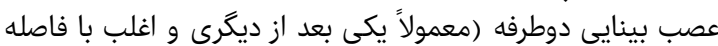

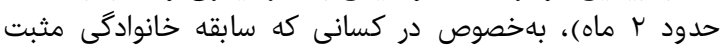
دارند، بايستى مد نظر قرار گيرد.

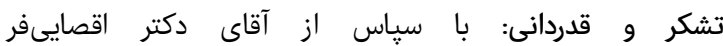
(نروافتالمولوزيست، بيمارستان فارابى، تهران) كه در ارزيابىهاى

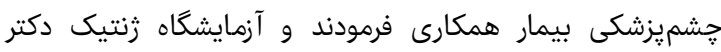
صنعتى كه با بررسى زنتيكى تشخيص بيمارى رإى را تاييد نمودند.

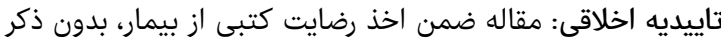

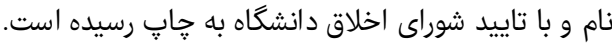
تعارض منافع: وجود ندارد.

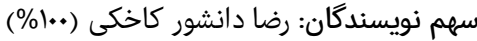


منابع

1- Leber Th. Ueber hereditäre und congenital-angelegte Sehnervenleiden. Graefes Arch Clin Exp Ophthalmol. 1871;17(2):249-91. [Germany]

2- Chinnery PF, Johnson MA, Wardell TM, Singh-Kler R, Hayes C, Brown DT, et al. The epidemiology of pathogenic mitochondrial DNA mutations. Ann Neurol. 2000;48(2):188-93.

3- Harding AE, Sweeney MG, Govan GG, Riordan-Eva P. Pedigree analysis in Leber hereditary optic neuropathy 